\title{
Penser par soi-même ou la question du maître vue par les encyclopédistes et par Kant
}

\section{Penser par soi-même}

Comment le mot d'ordre des encyclopédistes de penser par soi-même se conjugue-til avec la question de l'éducation qu'on pourrait formuler ainsi : l'homme a-t-il besoin d'un maitre ? Les encyclopédistes posent la question et aussitôt la reformulent selon les deux sens que l'on peut donner à la notion de maître. Le latin fournit deux termes qui recouvrent la notion de maître en français : dominus (celui qui possède les terres et qui domine les gens qui vivent sur ses terres : le seigneur) et magister (celui qui enseigne les connaissances et les règles : l'éducateur). Le dominus tient son autorité de ses titres de propriété, le magister de son savoir et de son éducation.

\subsection{La réponse exemplaire des encyclopédistes à la question du maître}

Dans quelle mesure les encyclopédistes ont-ils apporté une réponse exemplaire à la question du maître ? En ce qu'ils incitent le lecteur à substituer au premier sens du maître (dominus) le second (magister) : ils incitent le lecteur à ne pas céder à l'argument d'autorité fondé sur la domination mais à apprendre à penser par soi-même. Cette incitation requiert de mettre en place des dispositifs critiques de l'autorité fondée sur la domination, qui doivent à la fois échapper à la censure mais être lisibles pour le lecteur afin qu'il développe son esprit critique, d’où l'intérêt des réseaux d'articles porteurs de problématiques construites à partir des renvois, dans l'Encyclopédie.

- Véronique Le Ru - professeure des Universités en philosophie à l'Université de Reims CIRLEP EA 4299, UFR LSF, 57 rue Pierre Tainttinger BP 3051571 Reims cedex France, directrice du département de philosophie. Adresse de correspondance : leru@club-internet.fr

ORCID iD : https://orcid.org/0000-0003-2460-4506 
Les encyclopédistes, lecteurs avertis des Essais de Montaigne, et donc du Discours de la servitude volontaire de La Boétie (qui, en 1727, fut inséré dans la plupart des éditions des Essais) et enfin du Léviathan de Hobbes, connaissent bien les enjeux politiques de la question du maitre. Dans une perspective politique, la question posée peut se formuler en ces termes : l'homme peut-il vivre parmi ses semblables sans avoir besoin d'être inféodé à un seigneur?

\subsection{La position de Rousseau}

Rappelons la position de Rousseau : dans le Contrat social publié en 1762, il propose un pacte non plus de soumission mais d'association où les hommes trouvent intérêt à aliéner leur liberté dans la volonté générale représentée par le souverain. Subsiste alors la difficulté de concevoir ce souverain : est-il réellement l'expression de la volonté générale ? Ou bien, s'il est incarné par un individu, n’est-il pas doté d'une volonté particulière qui entre en conflit avec la volonté générale ? Rousseau résout le paradoxe selon lequel l'homme, à l'état civil, est un animal qui a besoin d'un maître qui, s'il est homme, a besoin d'un maitre, en distinguant le chef du gouvernement qui a la faillibilité et la faiblesse inhérentes à la condition humaine, et le souverain ou le corps politique institué par le pacte d'association en un être moral collectif représentant la volonté générale. Autrement dit, il écarte, dans le Contrat social, le risque encore présent dans son article ÉCONOMIE de voir le souverain poursuivre des fins propres au détriment de l'intérêt général et devenir un despote. Enfin, en faisant du souverain un être public et non un être humain, il rompt non seulement le cercle du maître résumé par Kant (voir la sixième proposition de l'Idée d'une Histoire universelle au point de vue cosmopolitique dans Kant, 1985, t. 2, p. 195), mais il garantit aussi qu'au regard du souverain conçu comme personne morale publique, tous les intérêts privés s’annulent. Mais cette résolution démocratique du contrat social est fort éloignée des encyclopédistes qui restent pour la plupart des monarchistes convaincus par le régime de monarchie parlementaire de l'Angleterre, qu'ils considèrent comme la meilleure forme de gouvernement.

Néanmoins, les encyclopédistes partagent avec Rousseau la conception de l'homme comme perfectible (voir Rousseau, 1971, p. 171-172)1, ce qui les conduit eux aussi au constat que l'homme a besoin d'un maitre mais entendu cette fois au sens d'éducateur (magister). Comment résoudre alors le conflit entre le mot d'ordre penser par soi-même et ce constat ? Et comment éviter la pétition de principe de léducation qui ne peut devenir meilleure qu'à condition de l'être déjà (puisqu'elle est donnée par un éducateur éduqué par l'éducation antérieure) ou, en d'autres termes, comment éviter la régression à l’infini dans la recherche de l'éducateur éduqué qui soit

1. Les encyclopédistes restreignent cette perfectibilité au domaine des connaissances et non au domaine moral. En effet, contrairement à ce qu'on dit parfois, les encyclopédistes sont loin d'être des chantres du progrès : si les sciences indéniablement progressent, il n'en va pas de même de la conscience morale : la plupart du temps, celle-ci est régie uniquement par l'intérêt. 
cependant un homme ? En effet, si l'homme a besoin d'un éducateur, cet éducateur, s'il est un homme, a aussi besoin d'un éducateur, etc.

De même que Rousseau brise, avant Kant, le cercle du maitre qui, s'il est un homme, est un animal qui a besoin d'un maître, de même les encyclopédistes résolvent, avant Kant, le paradoxe de l'éducateur qui, s'il est un homme, a aussi besoin d'un éducateur.

\subsection{Le cercle du maître ou de l'éducateur éduqué}

Dans ses Réflexions sur l'éducation, Kant prend acte du problème de l'éducateur en disant que c'est le problème le plus difficile qui soit. En effet, pour briser le cercle, soit on pose qu'il puisse y avoir un éducateur qui ne soit pas un homme - un Dieu omniscient, par exemple - qui viendrait éclairer les hommes (mais Kant rejette cette solution par son refus d'une intervention divine directe) ; soit on pose qu'il y a une instance s'éclairant elle-même dans le processus éducatif. C'est cette solution que développe Kant en considérant l'éducation non sur le plan de l'individu mais sur le plan interindividuel de l'espèce humaine. Celle-ci permet de penser, par les rapports individuels qui la constituent et par sa pérennité, l'accomplissement des progrès de la raison humaine dans le public et dans le temps. Le public composé des rapports interindividuels finit par séclairer (voir Kant, 1991, p. 44 : « qu'un public s’éclaire luimême est plus probable ; cela est même inévitable pourvu qu'on lui accorde la liberté ») surtout si on pense ses progrès non dans la mesure d'une vie individuelle mais dans celle d'une succession de générations.

\section{La solution des encyclopédistes au problème de l'éducateur éduqué}

En quoi les encyclopédistes apportent-ils une réponse convaincante, avant Kant, à la question de l'éducation ? Parce qu'ils ont fait le choix, comme Kant, de penser le problème de l'éducation non sur le plan de l'individu mais sur le plan des rapports interindividuels. Ils assument ce choix, dans le sens repris par Kant, du public qui finit par s'éclairer quand on pense ses progrès à l'échelle des neveux. Mais ils l'assument aussi dans un sens tout à fait original qui est celui de l'éducateur : en effet, l'éducateur de l'Encyclopédie est également composé de rapports interindividuels. Léducateur est un auteur multiple : la société de gens de lettres qui, comme son nom l'indique, fait société et, de ce fait, acquiert également un statut d'être public. Aussi bien d'Alembert que Diderot insistent, dans les deux articles ÉLÉMENTS DES SCIENCES et ENCYCLOPÉDIE où les deux éditeurs définissent respectivement l'Encyclopédie, sur le fait que cet ouvrage ne peut être l'entreprise d'un seul homme mais que sa richesse tient précisément à sa multiplicité d’auteurs. D’Alembert, dans l'article ÉLÉMENTS DES SCIENCES, montre que ni l'élève récemment instruit ni le génie passé maître dans une science ou dans un art ne sont aptes à enseigner les éléments d'une science ou d'un art. L'élève même éclairé ne peut devenir un éducateur 
par son incompétence à saisir les principes ou les éléments de la science ou de l'art qu'il pratique (saisie qui demande une connaissance approfondie de cette science ou de cet art (voir l'article ÉLÉMENTS DES SCIENCES de l'Encyclopédie dans 1755, t. V, p. 496)) et le maitre pense davantage à inventer qu'à enseigner parce que son génie lui fait préférer la gloire et la postérité à l'éducation. C'est pourquoi l'exposé encyclopédique des éléments des sciences et des arts ne peut être le fait que d'une société de gens de lettres, chacun versé dans l'art ou la science qu'il expose, mais tous unis dans l'exigence, pour l'intérêt du genre humain et pour les progrès des connaissances, de les présenter clairement au plus grand nombre.

\subsection{Le refus de l'argument d'autorité}

Le danger que les encyclopédistes veulent éviter, dans l'éducation qu'ils proposent, c'est celui de l'argument d'autorité qui repose sur l'imposture et non sur le savoir. Il est remarquable, en ce sens, que l'une des premières occurrences du mot d'ordre penser par soi-même dans l'Encyclopédie se situe précisément dans l'article de Diderot intitulé AUTORITÉ dans les discours et dans les écrits. Diderot dénonce, dans l'usage de l'argument d'autorité et dans l'abus des citations, un exercice démagogique destiné " à éblouir le peuple, à tromper les petits esprits et fournir du babil aux demi-savants " (voir l'article AUTORITÉ dans les discours et dans les écrits de l'Encyclopédie dans 1751, t. I, p. 900). Et il ajoute : «Ceux à qui il manque assez d'étendue dans l'esprit pour penser eux-mêmes, se contentent des pensées d'autrui, et comptent les suffrages » (1751, t. I, p. 900). Toute la suite de l'article est une critique en règle de l'autorité qui usurpe les droits de la raison dans l'éducation. Diderot file la métaphore de l’aveugle développée par Descartes dans la Lettre-préface des Principes de la philosophie. Quand Descartes dit : " il vaut beaucoup mieux se servir de ses propres yeux pour se conduire $[\ldots]$ que non pas de les avoir fermés et suivre la conduite d'un autre " (1963-1973, t. 3, p. 771), Diderot reprend : «Ceux qui se conduisent dans leurs études par l'autorité seule, ressemblent assez à des aveugles qui marchent sous la conduite d'autrui " (voir l'article AUTORITÉ dans les discours et dans les écrits dans 1751, t. I, p. 901). Et Diderot conclut en disant : « Je me représente les esprits qui ne veulent rien devoir à leurs propres réflexions, et qui se guident sans cesse d'après les idées des autres, comme des enfants dont les jambes ne s'affermissent point, ou des malades qui ne sortent point de l'état de convalescence, et ne feront jamais un pas sans un bras étranger " (voir l'article AUTORITÉ dans les discours et dans les écrits dans 1751, t. I, p. 901). Ces termes sont extrêmement proches de ceux que Kant utilisera trente ans plus tard dans sa Réponse à la question Qu'est-ce que les Lumières ?² ou dans l’Anthropologie du point de vue pragmatique (voir $\$ 48$ et $\$ 59$ notamment).

2. Kant parle notamment de la roulette d'enfant qui permet de ne pas apprendre à marcher. 


\subsection{Penser par soi-même plutôt que penser par son maître}

Dans l'article AUTORITÉ, Diderot fait de l'exigence de penser par soi-même le problème central de l'éducation. Au mieux l'autorité peut-elle servir à nous soutenir en cas de faiblesse dans le chemin que la raison nous montre, en aucun cas elle ne peut servir à nous conduire. Dans l'article ARISTOTÉLISME qui est anonyme, le propos est le même : si la philosophie a eu son Descartes, il faut espérer que la nature donnera à la société ce qu'elle a déjà donné à la philosophie ; alors "la société aura son Descartes qui renversera une infinité de préjugés et fera rire nos derniers neveux de toutes les sottises que nous avons adoptées " (voir l'article ARISTOTÉLISME de l'Encyclopédie dans 1751, t. I, p. 670). Cependant, l'article insiste sur la difficulté que Descartes a rencontrée pour imposer l'évidence de la raison : "Par la méthode qu'on suivait alors, il était impossible qu'on sortît de la barbarie; on ne raisonnait pas pour découvrir de nouvelles vérités, on se contentait de savoir ce qu'Aristote avait pensé » (p. 665). Et, à la fin de l'article, apparaît de nouveau la métaphore de l'aveugle doublée du problème de Molyneux : de même qu'un aveugle qui recouvre la vue est désorienté, ne sait plus se repérer et doit apprendre à voir, de même celui qui ne pense pas mais qui a un maître à penser [Aristote] à qui l'on [Descartes] ôte le bandeau des préjugés commence par être ébloui par tant de lumière et doit apprendre à penser. Cette allusion au problème de Molyneux pourrait désigner Diderot comme l'auteur de l'article, d'autant qu'il vient de publier la Lettre sur les aveugles ${ }^{3}$ et qu'il a la responsabilité de la plupart des articles concernant l'histoire de la philosophie. Le fait que dans la Lettre sur les aveugles, comme dans l'article ARISTOTÉLISME, la référence à Descartes soit présentée de manière très positive (sur la référence de Diderot à Descartes, voir Le Ru, 2000) rend cette hypothèse encore plus plausible : à l'époque, entre 1749 et 1751, Diderot ne cachait pas son admiration pour Descartes. Ce dernier était-il pour autant un maître à penser pour Diderot ? Certainement pas, Diderot avait déjà pris ses distances vis-à-vis de la philosophie de Descartes et de la distinction des substances. Mais Descartes reste, pour lui, un modèle de libération de la superstition et des préjugés : le mot d'ordre de penser par soi-même prend racine dans l'entreprise hardie du doute cartésien et de toute sa philosophie qui lève, avant Réaumur, la cataracte du lecteur : en ce sens, on peut lire la reprise de la métaphore de l'aveugle comme un hommage à Descartes. A vrai dire, le rapport de Diderot à Descartes est le même que celui qu'il entretient avec l'histoire de la philosophie en général et qu'il définit parfaitement dans son article ÉCLECTISME : «L'éclectique est un philosophe qui foulant aux pieds le préjugé, la tradition, l'ancienneté, le consentement univer-

3. Diderot y soutient, contrairement à Condillac, que l'aveugle-né opéré ne peut identifier par la vue seule l'objet qu'il identifiait par le toucher. Son expérience passée du toucher ne l'aide en rien à voir tant qu'il n'apprend pas à comparer ses sensations visuelles et tactiles lors d'expériences sensibles répétées. Diderot ajoute que cet apprentissage se fera diversement selon les individus et leur éducation. Sur cette question dans la Lettre sur les aveugles, voir «Lettre sur les aveugles". (2000). Recherches sur Diderot et sur l'Encyclopédie, 28. 
sel, l'autorité, en un mot tout ce qui subjugue la foule des esprits, ose penser de luimême, remonter aux principes généraux les plus clairs, les examiner, les discuter, n'admettre rien que sur le témoignage de son expérience et de sa raison " (voir l'article ÉCLECTISME de l'Encyclopédie dans 1755, t. V, p. 270).

\section{L'intérêt pour le genre humain}

C'est l'intérêt pour le genre humain qui conduit l'éclectique à transmettre sa récolte, c'est-à-dire l'éducation qu'il s'est forgée à partir des philosophies qu'il a étudiées : "Ce n'est point un homme qui plante ou qui sème ; c'est un homme qui recueille et qui crible. Il jouirait tranquillement de la récolte qu'il aurait faite, il vivrait heureux, et mourrait ignoré, si l'enthousiasme, la vanité, ou peut-être un sentiment plus noble, ne le faisait sortir de son caractère " (voir l'article ÉCLECTISME de l'Encyclopédie dans 1755, t. V, p. 270). C'est en lisant ceux qui ont choisi de penser par eux-mêmes qu'on apprend à penser par soi-même ${ }^{4}$. D'Alembert, lui aussi, dans l'article ÉRUDITION, souligne toutes les lumières que l'on peut puiser des lectures des philosophes si l'on a appris à penser soi-même, c'est-à-dire si l'on est soi-même philosophe ${ }^{5}$. Enfin, Jaucourt, dans la composition de ses dix-sept mille articles ${ }^{6}$, ne cesse de mettre en œuvre la méthode de l'éclectique en lisant, en écrivant.

Diderot, lui aussi, insiste, dans l'article ENCYCLOPÉDIE, sur le fait que l'Encyclopédie ne peut être l'ouvrage d'un seul homme et qu'il ne peut s'exécuter que " par une société de gens de lettres et d'artistes, épars, occupés chacun de sa partie, et liés seulement par l'intérêt du genre humain, et par un sentiment de bienveillance réciproque $~^{7}$. Dans le même article, Diderot présente le système des renvois comme la doublure critique de l'Encyclopédie : elle « opère très promptement sur les bons esprits, et elle opère infailliblement et sans aucune conséquence fâcheuse, secrètement

4. Parmi les auteurs modernes « jaloux de la prérogative la plus belle de l'humanité, la liberté de penser par soi-même ", Diderot mentionne bien sûr Descartes mais aussi Jordanus Brunus, François Bacon, Campanella, Hobbes, Leibniz, Malebranche (voir l'article ÉCLECTISME dans 1755, t. V, p. 283).

5. Voir l'article ÉRUDITION de l'Encyclopédie dans 1755, t. V, p. 918 : «On peut sans doute savoir l'histoire des pensées des hommes sans penser soi-même ; mais un philosophe peut lire avec beaucoup d'utilité le détail des opinions de ses semblables ; il y trouvera souvent des germes d'idées précieuses à développer, des conjectures à vérifier, des faits à éclaircir, des hypothèses à confirmer ».

6. Nous faisons référence ici au sous-titre de l'ouvrage collectif dirigé par Gilles Barroux et François Pépin, Le chevalier de Jaucourt - l'homme aux dix-sept mille articles, 2015. L'Encyclopédie comportant environ 74000 articles, cela signifie que Jaucourt en a rédigé presque un quart.

7. Voir l'article ENCYCLOPÉDIE de l'Encyclopédie dans 1755, t. V, p. 636 et Diderot ajoute : « Je dis une société de gens de lettres et d'artistes, afin de rassembler tous les talents. Je les veux épars, parce qu'il n'y a aucune société subsistante d'où l'on puisse tirer toutes les connaissances dont on a besoin [...] J'ajoute, des hommes liés par l'intérêt général du genre humain et par un sentiment de bienveillance réciproque, parce que ces motifs étant les plus honnêtes qui puissent animer des âmes bien nées, ce sont aussi les plus durables». 
et sans éclat, sur tous les esprits " (voir l'article ENCYCLOPÉDIE dans 1755, t. V, p. 643). Si on utilise bien la fonction critique des renvois de choses, avec le temps, tous les préjugés seront renversés et tous les esprits éclairés (p. 643 : «Il y aurait un grand art et un avantage infini dans ces derniers renvois. L'ouvrage entier en recevrait une force interne et une utilité secrète, dont les effets sourds seraient nécessairement sensibles avec le temps »). L'enjeu de l'ouvrage n'est pas seulement critique, il est aussi éducatif. En ce sens, la fin de l'Encyclopédie, c'est sa fin : une fois les lecteurs éduqués, une fois la révolution dans les esprits accomplie, elle aura achevé sa mission d'instruire le public (p. 643 : « Aujourd'hui que la philosophie s'avance à grands pas ; qu'elle soumet à son empire tous les objets de son ressort; que son ton est le ton dominant, et qu'on commence à secouer le joug de l'autorité et de l'exemple pour s'en tenir aux lois de la raison, il n'y a presque pas un ouvrage élémentaire et dogmatique dont on soit entièrement satisfait »). En effet, la fonction critique des renvois doit s'accompagner d'un enseignement positif des éléments des sciences et des arts. Les éléments bien faits doivent apprendre à penser par soi-même ${ }^{8}$ et doivent œuvrer aux progrès et à la gloire des sciences (p. 496 : «à leur progrès, parce qu'en facilitant aux génies heureux l'étude de ce qui est connu, on les met en état d'y ajouter davantage et plus promptement ; à leur gloire, parce qu'en les mettant à la portée d'un plus grand nombre de personnes, on se procure un plus grand nombre de juges éclairés »). Les encyclopédistes ont une conscience aiguë de travailler pour la postérité et l'intérêt du genre humain afin que le public finisse par séclairer et devienne ainsi plus vertueux et plus heureux 9 . Le public, ce sont, pour les encyclopédistes, les lecteurs de l'Encyclopédie, et les éducateurs éclairés, ce sont eux-mêmes en tant qu'ils forment une société de gens de lettres et non simplement une sommation d'individus. Diderot aussi bien que d'Alembert mettent l'accent sur l'intérêt pour le genre humain qui unit leur société et donne sens à leur entreprise.

8. Voir l'article ÉLÉMENTS DES SCIENCES dans 1755, t. V, p. 496 : « Nous n'ajouterons plus qu'un mot sur la manière d'étudier quelques sortes d'éléments que ce puisse être, en supposant ces éléments bien faits. Ce n'est point avec le secours d'un maitre qu'on peut remplir cet objet, mais avec beaucoup de méditation et de travail. Savoir des éléments, ce n'est pas seulement connaitre ce qu'ils contiennent, c'est en connaître l'usage, les applications, et les conséquences ; c’est pénétrer dans le génie de l'inventeur, c'est se mettre en état d'aller plus loin que lui, et voilà ce qu'on ne fait bien qu'à force d'étude et d'exercice : voilà pourquoi on ne saura jamais parfaitement que ce qu'on a appris soi-même [...] Le propre d'un bon livre d'éléments est de laisser beaucoup à penser ".

9. Voir l'article ENCYCLOPÉDIE dans 1755, t. V, p. 635 : «En effet, le but d'une Encyclopédie est de rassembler les connaissances éparses sur la surface de la terre, d'en exposer le système général aux hommes avec qui nous vivons, et de le transmettre aux hommes qui viendront après nous; afin que les travaux des siècles passés n'aient pas été des travaux inutiles pour les siècles qui succéderont; que nos neveux, devenant plus instruits, deviennent en même temps plus vertueux et plus heureux, et que nous ne mourions pas sans avoir bien mérité du genre humain ». 


\section{La réponse kantienne à la question de l'éducation}

La réponse kantienne à la question de l'éducation a un air de famille avec celle que proposent les encyclopédistes. En effet, Kant insiste lui aussi que le fait ce sont les rapports interindividuels et intergénérationnels des élèves qui font que le public finit par s'éclairer. Dans La réponse à la question : qu'est-ce que les Lumières?, il souligne que s'il est difficile pour un individu de s'arracher à l'état de tutelle qui est devenu pour lui une seconde nature, en revanche si l'on pense le problème de l'éducation sur le plan interindividuel et intergénérationnel, alors le public finit toujours par s'éclairer : «Car il se trouvera toujours quelques êtres pensant par eux-mêmes, même parmi les tuteurs en exercice du plus grand nombre, pour rejeter eux-mêmes le joug de l'état de tutelle et pour propager ensuite autour d'eux l'esprit d'une appréciation raisonnable de la propre valeur et de la vocation de tout homme à penser par soi-même " (Kant, 1991, p. 44).

Mais alors que les encyclopédistes posaient collectivement en amont et en aval, si l'on peut dire, la question de l'éducation, Kant ne la pose qu'en aval : c'est seulement dans la réception de l'éducation que l'on doit penser les rapports interindividuels et intergénérationnels. Les encyclopédistes, en ce sens, vont plus loin que Kant car ils posent aussi collectivement la question de l'éducateur : l'éducateur n'est pas un individu mais une société de gens de lettres, l'éducateur n'est pas un maître qui aurait une autorité sur ses élèves mais l'éducateur est un auteur multiple, composé de rapports interindividuels et intergénérationnels : entre Montesquieu et Voltaire d'un côté, d'Alembert, Diderot, Rousseau de l'autre, il y a une génération d'écart, sans compter les nombreuses références des encyclopédistes aux auteurs qui les ont précédés.

\section{La signification kantienne de l'expression penser par soi-même}

Dans sa Réponse à la question : quest-ce que les Lumières ?, Kant reprend la corrélation instaurée par les encyclopédistes entre l'exigence de penser par soi-même et la question de l'éducation. En effet, on l'a vu à travers les articles AUTORITÉ et ARISTOTÉLISME, l'exigence de penser par soi-même est corrélée au refus de l'argument d'autorité : refuser l'autorité d'un seul maître ou tuteur signifie aussi lire avec attention les auteurs pour cribler le meilleur de leurs ouvrages pour s'instruire et forger sa conscience morale et politique. Le futur éduqué doit être éclectique pour devenir éduqué. Cette solution de répondre à l'exigence de penser par soi-même par le refus de l'autorité et l'éclectisme n'est pas celle de Kant. Pour ce dernier, ce sont certains des tuteurs eux-mêmes qui tôt ou tard rejettent le joug qu'ils infligent à leurs élèves : "Car il se trouvera toujours quelques êtres pensant par eux-mêmes, même parmi les tuteurs en exercice du plus grand nombre, pour rejeter eux-mêmes le joug 
de l'état de tutelle " (Kant, 1991, p. 44). L'état de tutelle n'explose pas par l'éduqué, il implose par l’éducateur.

On comprend mieux alors pourquoi Kant considère que l'expression Sapere aude est la devise des Lumières ${ }^{10}$, mais que cette injonction à penser par soi-même est à lire comme un moment surtout critique : il s'agit de secouer le joug de la superstition et de sortir de l'état de tutelle dont on est soi-même responsable. En ce sens, il faut joindre à ce moment les deux autres moments représentés par les deux autres maximes du sens commun, comme Kant le propose dans le paragraphe 40 de la Critique de la Faculté de Juger, où il analyse l'expression penser par soi-même en trois maximes. Penser par soi-même, pour Kant, c'est conquérir sa majorité rationnelle, ce qui signifie se soustraire au principe d'autorité pour reconnaître une communauté que lie la raison comme adresse à tous et à chacun. Pour présenter cette conception de l'exercice de la raison comme déploiement de la liberté, Kant expose les trois maximes du sens commun an tant que normes de l'usage d'un entendement qui s'élève à l'universel : 1) Penser par soi-même 2) Penser en se mettant à la place de tout autre 3) Toujours penser en accord avec soi-même. Les trois maximes s'articulent dans leur réciprocité et indissociabilité. Penser par soi-même, maxime de la pensée sans préjugé, moment que Kant qualifie de l'entendement, est celle d'une raison qui n'est jamais passive (le préjugé étant défini par Kant comme la tendance à la passivité et, par conséquent, à l'hétéronomie de la raison). Kant dénonce, chemin faisant, la superstition comme le préjugé le plus grand parce qu'il « consiste à se représenter la nature comme n'étant pas soumise aux règles que l'entendement de par sa propre et essentielle loi lui donne pour fondement " (Kant, 1984, p. 128). Et il ajoute que les Lumières opèrent la libération de la superstition. Cette première maxime implique l'ouverture de sa pensée en se mettant à la place de tout autre, c'est-à-dire la deuxième maxime qui est celle de l'adoption du point de vue universel (moment que Kant désigne comme celui de la faculté de juger). En ce sens, penser par soi-même ne signifie en rien s'enfermer dans son ego, dans un solipsisme où sa pensée serait le seul îlot de réalité mais, au contraire, penser par soi-même veut dire se mettre à l'abri des tentations de connaître ce qui dépasse l'entendement, en se donnant sans cesse sa propre loi qui est aussi celle de tous. Autrement dit, penser par soi-même ne signifie pas s'isoler du monde mais constitue un moment négatif où l'on rejette une aspiration qui est aussi commune à tous les hommes, à savoir l'aspiration à connaître ce qui dépasse l'entendement (moment du rejet de la superstition). Une fois que l'on a bien saisi la portée de ce moment critique ou négatif, on comprend que la première maxime ne se referme pas sur l'ego mais qu'elle ouvre au contraire sur l'alter ego et sur l'adoption du point de vue universel : penser en se mettant à la place de tout autre. De cette deuxième maxime qui signifie l'usage de l'autonomie intellectuelle et l'accès à la majorité rationnelle, résulte la troisième

10. Kant, 1991, p. 43 : « Sapere aude! Aie le courage de te servir de ton propre entendement ! Voilà la devise des Lumières ». 
maxime, à savoir toujours penser en accord avec soi-même. Cette troisième maxime est celle de la manière de penser conséquente, moment de la raison, le plus difficile à mettre en œuvre : «on ne le peut qu'en liant les deux premières maximes et après avoir acquis une maîtrise rendue parfait par un exercice répété» (Kant, 1984, p. 128).

La liaison des deux premières maximes à la troisième interdit de s'en tenir à l'apparence de leur juxtaposition. Car l'unité articulée de ces trois maximes du sens commun définit le sens de l'acte de philosopher : "Être conséquent, c'est la première obligation d'un philosophe, et c'est pourtant celle à laquelle on se conforme le plus rarement. Les anciennes écoles grecques nous en donnent plus d'exemples, que ne nous en offre notre siècle syncrétique, où l'on se forme avec des principes contradictoires, un certain système composite, plein de mauvaise foi et de frivolité, parce que cela convient mieux à un public qui est content de savoir un peu de tout, sans rien savoir en somme, et d'être propre à tout » (Kant, 1943, p. 23).

\section{Conclusion}

Penser par soi-même signifie, aussi bien pour les encyclopédistes que pour Kant, d'abord se libérer des chaînes de la superstition et du joug d'un maître, pour accéder au point de vue de l'universel et se mettre en condition de philosopher. Philosopher c'est toujours adopter le point de vue de l'universel, en luttant contre le relativisme des valeurs et les intérêts individuels (y compris les siens). C'est ainsi en adoptant toujours le point de vue de l'universel qu'on est conséquent avec soi-même et qu'on est son propre maître : d'éduqué, on est devenu éducateur de soi-même. Si Kant approfondit l'activité de penser par soi-même comme accès à l'universel, il puise cette réflexion dans les Lumières et l'Encyclopédie de Diderot et de d'Alembert car ce sont les encyclopédistes, les premiers, qui ont montré et mis en œuvre la corrélation entre l'exigence de penser par soi-même et l'accomplissement de l'éducation. En ce sens, les encyclopédistes nous apprennent deux choses essentielles. Premièrement, l'éducation est perfectible à condition que l'éducateur et l'éduqué soient compris non dans un rapport individuel qui risque de se transformer en un rapport d'autorité mais dans des rapports interindividuels. Deuxièmement, le modèle de l'Encyclopédie est un modèle éducatif : sa péremption garantit sa réussite. De même que la fin d'une Encyclopédie particulière, c'est sa fin, de même la fin d'une éducation donnée c'est sa fin.

\section{RÉFÉRENCES}

Barroux, G., Pepin, F. (dir.). (2015). Le chevalier de Jaucourt - l'homme aux dix-sept mille articles. Paris : Société Diderot.

Descartes, R. (1963-1973). Euvres philosophiques. Tomes 1-3. Paris : Garnier. 
Diderot, D., Alembert, J. de. (1751-1765 vol. de textes ; 1762-1772 vol. de planches ; 1766-1780 vol. de suppléments et de tables ). Encyclopédie ou dictionnaire raisonné des sciences, des arts et des métiers. Paris : Briasson, David, Le Breton et Durand (vol. de textes et planches), puis Paris : Panckoucke et Amsterdam : Rey (vol. de suppléments et de tables).

Kant, E. (1985). Euvres philosophiques. Paris : Gallimard.

Kant, E. (1991). Réponse à la question : qu'est-ce que les Lumières? (J.-F. Poirier et F. Proust, trad.). Paris : GF.

Kant, E. (1984). Critique de la Faculté de Juger. (A. Philonenko, trad.). Paris : Vrin.

Kant, E. (1943). Critique de la Raison Pratique. (F. Picavet, trad.). Paris : PUF.

Le Ru, V. (2000). La Lettre sur les aveugles et le bâton de la raison. Recherches sur Diderot et sur l'Encyclopédie, 28, 25-41.

Rousseau, J.-J. (1971). Discours sur l'origine de l'inégalité. Paris : GF.

RÉSUMÉ : Comment le mot d'ordre des encyclopédistes de penser par soi-même se conjugue-t-il avec la question de l'éducation qu'on pourrait formuler ainsi : l'homme a-t-il besoin d'un maître ? Les encyclopédistes posent la question et aussitôt la reformulent selon les deux sens que l'on peut donner à la notion de maître. Le latin fournit deux termes qui recouvrent la notion de maître en français : dominus (celui qui possède les terres et qui domine les gens qui vivent sur ses terres : le seigneur) et magister (celui qui enseigne les connaissances et les règles : l'éducateur). Le dominus tient son autorité de ses titres de propriété, le magister de son savoir et de son éducation. Nous comparerons la solution que donnent les encyclopédistes à la question de l'éducation à celle que propose Kant, nous comparerons également la signification respective qu'ils donnent à l'exigence de penser par soi-même et nous montrerons que l'injonction de penser par soi-même est corrélée à celle de l'éducation aussi bien chez les encyclopédistes que chez Kant.

Mots-clés : Encyclopédistes, éducation, penser par soi-même, Jean Le Rond d'Alembert, Denis Diderot, Emmanuel Kant

\section{To think by oneself or the question of the master in the thought of the Encyclopédistes and of Kant}

ABSTRACT: How does the Encyclopédistes' watchword 'to think for oneself' relate to the question of education, which could be formulated as: does man need a master? As soon as the Encyclopédistes ask the question, they propose a new way of thinking about the two meanings which can be given to the notion of master: dominus ("the lord"), and magister ("the teacher"). The lord has authority thanks to his status as a land owner or castle owner, the teacher is in a position of power by virtue of his knowledge and education. The Encyclopédistes' answer to the question concerning 
education will be compared to that given by Kant, and the meaning the Encyclopédistes assign to the expression 'to think for oneself' will also be juxtaposed with Kant's interpretation. In this way, the correlation between education and 'thinking for oneself' observed by both Kant and the Encyclopédistes will be demonstrated.

Keywords: Encyclopédistes, education, thinking for oneself, Jean Le Rond d'Alembert, Denis Diderot, Emmanuel Kant 